\title{
Visit End Time
}

National Cancer Institute

\section{Source}

National Cancer Institute. Visit End Time. NCI Thesaurus. Code C102558.

The stop time of the visit. 